\title{
Correction to: Complex patterns of genetic and morphological differentiation in the Smallmouth Bass subspecies (Micropterus dolomieu dolomieu and M. d. velox) of the Central Interior Highlands
}

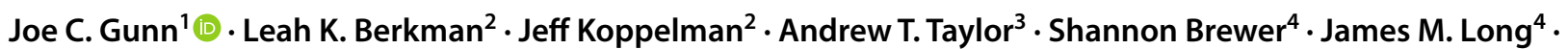
Lori S. Eggert ${ }^{1}$

Published online: 24 August 2020

(c) Springer Nature B.V. 2020

\section{Correction to: Conservation Genetics}

https://doi.org/10.1007/s10592-020-01295-1

In the original publication of the article, Table 2 was published incorrectly. The correct Table 2 is given in this Correction.

The original article can be found online at https://doi.org/10.1007/ s10592-020-01295-1.

Joe C. Gunn

jcg5g9@mail.missouri.edu

1 Division of Biological Sciences, University of Missouri, 226

Tucker Hall, Columbia, MO 65211, USA

2 Missouri Department of Conservation, 3500 E. Gans Rd., Columbia, MO 65201, USA

3 Department of Natural Resource Ecology and Management, Oklahoma State University, Stillwater, OK 74078, USA

4 U.S. Geological Survey, Oklahoma Cooperative Fish and Wildlife Research Unit, Department of Natural Resource Ecology and Management, Oklahoma State University, Stillwater, OK 74078, USA 
Table 2 Raw and adjusted trait means of standard length (SL), orbital length (OL), head length (HL), and body depth (BD) for Neosho and Northern Smallmouth Bass samples assessed by (a) taxonomic range, and (b) genetic assignment

\begin{tabular}{|c|c|c|c|c|c|c|c|c|}
\hline \multirow[t]{2}{*}{ Trait } & \multicolumn{2}{|c|}{ Raw mean $(\mathrm{mm})$} & \multicolumn{2}{|c|}{ Adjusted mean (mm) } & \multicolumn{4}{|c|}{ ANCOVA results } \\
\hline & Neosho & Northern & Neosho & Northern & $F_{\text {int }}$ & $F_{\text {cat }}$ & $p_{\text {int }}$ & $p_{\text {cat }}$ \\
\hline \multicolumn{9}{|c|}{ (a) Taxonomic range } \\
\hline SL & 234.864 & 246.726 & 232.274 & 238.232 & 1.200 & 2.100 & 0.271 & 0.166 \\
\hline OL & 14.135 & 14.474 & 14.060 & 14.322 & 30.628 & 0.110 & $<0.001$ & 0.744 \\
\hline $\mathrm{HL}$ & 79.966 & 76.463 & 79.616 & 73.282 & 0.220 & 14.070 & 0.636 & 0.001 \\
\hline $\mathrm{BD}$ & 66.803 & 68.340 & 65.766 & 65.013 & 1.130 & 0.160 & 0.289 & 0.696 \\
\hline \multicolumn{9}{|c|}{ (b) Genetic assignment } \\
\hline SL & 206.588 & 244.809 & 232.809 & 234.963 & 1.800 & 0.300 & 0.181 & 0.620 \\
\hline OL & 13.925 & 14.721 & 14.521 & 14.488 & 2.041 & 0.100 & 0.158 & 0.756 \\
\hline $\mathrm{HL}$ & 71.921 & 78.259 & 79.433 & 74.645 & 0.120 & 3.140 & 0.728 & 0.097 \\
\hline BD & 58.026 & 68.065 & 66.834 & 63.973 & 0.160 & 0.180 & 0.693 & 0.681 \\
\hline
\end{tabular}

Trait means were back-transformed to provide values in millimeters. ANCOVA results are based on coefficients calculated from log-transformed trait values and include $F$-statistics and $p$-values for interactions (int) and categories (cat; taxonomic range or genetic assignment)

Publisher's Note Springer Nature remains neutral with regard to jurisdictional claims in published maps and institutional affiliations. 